ARTICLE

Received 25 Jul 2012 | Accepted 2 Jan 2013 | Published 5 Feb 2013 DOl: 10.1038/ncomms2437

\title{
Magnetic field-induced dissipation-free state in superconducting nanostructures
}

\author{
R. Córdoba1,2, T.I. Baturina ${ }^{3,4}$, J. Sesé1,2, A. Yu Mironov³, J.M. De Teresa1,2,5, M.R. Ibarra1,2,5,

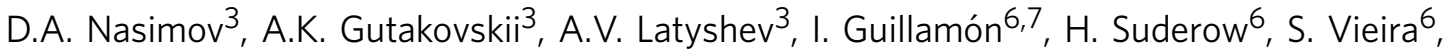 \\ M.R. Baklanov ${ }^{8}$, J.J. Palacios ${ }^{9}$ \& V.M. Vinokur ${ }^{4}$
}

A superconductor in a magnetic field acquires a finite electrical resistance caused by vortex motion. A quest to immobilize vortices and recover zero resistance at high fields made intense studies of vortex pinning one of the mainstreams of superconducting research. Yet, the decades of efforts resulted in a realization that even promising nanostructures, utilizing vortex matching, cannot withstand high vortex density at large magnetic fields. Here, we report a giant reentrance of vortex pinning induced by increasing magnetic field in a $\mathrm{W}$-based nanowire and a TiN-perforated film densely populated with vortices. We find an extended range of zero resistance with vortex motion arrested by self-induced collective traps. The latter emerge due to order parameter suppression by vortices confined in narrow constrictions by surface superconductivity. Our findings show that geometric restrictions can radically change magnetic properties of superconductors and reverse detrimental effects of magnetic field.

\footnotetext{
${ }^{1}$ Laboratorio de Microscopías Avanzadas, Instituto de Nanociencia de Aragón, Universidad de Zaragoza, Zaragoza E-50018, Spain. ${ }^{2}$ Departamento de Física de la Materia Condensada, Universidad de Zaragoza, Zaragoza 50009, Spain. ${ }^{3}$ A. V. Rzhanov Institute of Semiconductor Physics SB RAS, 13 Lavrentjev Avenue, Novosibirsk 630090, Russia. ${ }^{4}$ Materials Science Division, Argonne National Laboratory, Argonne, Illinois 60439, USA. ${ }^{5}$ Instituto de Ciencia de Materiales de Aragón, Universidad de Zaragoza-CSIC, Facultad de Ciencias, Zaragoza 50009, Spain. ${ }^{6}$ Laboratorio de Bajas Temperaturas, Departamento de Física de la Materia Condensada, Instituto de Ciencia de Materiales Nicolás Cabrera, Facultad de Ciencias, Universidad Autónoma de Madrid, Madrid E-28049, Spain. ${ }^{7}$ H.H. Wills Physics Laboratory, University of Bristol, Tyndall Avenue, Bristol BS8 1TL, UK. ${ }^{8}$ IMEC Kapeldreef 75, Leuven B-3001, Belgium. ${ }^{9}$ Departamento de Física de la Materia Condensada, Instituto de Ciencia de Materiales Nicolás Cabrera, Facultad de Ciencias, Universidad Autónoma de Madrid, Madrid E-28049, Spain. Correspondence and requests for materials should be addressed to H.S. (email: Hermann.suderow@uam.es).
} 
n 1936, Schubnikow et al. ${ }^{1,2}$ broke ground for applications of superconductors. He pointed out the vital distinction between type-I superconductors, in which currents flow only at the surface and superconductivity is destroyed by weak fields and a new type of superconductor, now called a type-II superconductor, capable of carrying bulk supercurrent at relatively high fields. The understanding that the behaviour of type-II superconductors is due to quantized magnetic vortices was achieved by Abrikosov ${ }^{3}$ in the 1957. Unluckily, the same vortices that stabilize a superconductor in magnetic fields, compromise its key characteristic, its ability of carrying electrical current with zero resistance. The vortices move in response to an electrical current, dissipating energy and destroying the zero-resistance state ${ }^{4}$. One of the central problems for applications of superconductivity is thus immobilizing vortices to ensure zero electrical power losses. Motivated by this quest, vortex pinning has been over decades the subject of extensive experimental and theoretical studies ${ }^{5-7}$. Generally, the feasibility of superconducting technology is proven. However, it is often desirable to work at temperatures and fields comparable to the critical values where superconductivity is lost. Then, pinning effects are dramatically reduced ${ }^{7}$. Yet there have been alluring communications of an enhancement of superconducting parameters by magnetic field and a negative magnetoresistance in thin superconducting wires $^{8-11}$. These observations however promising challenged our understanding, as they often referred to the field and temperature range where strong vortex pining were not expected.

Here we report a giant reentrance of superconductivity, that is, recovery of the dissipation-free state, by increasing perpendicular magnetic field in a thin wire and thin superconducting film patterned into an array of small holes. We observe suppression of the resistance over at least four orders of magnitude over a wide range of magnetic fields. The experiments are carried out at high magnetic fields and temperatures, where both the wire and the film are densely populated with vortices and where conventional pinning mechanisms are expected to fall ineffective. We demonstrate that this reentrant dissipation-free state forms as a result of constricting vortices into a narrow stripe (in a wire) or small cells (in a film) by surface superconductivity. The densely packed vortices collectively suppress the nearby order parameter thus generating deep potential wells for themselves and selfarresting their own motion.

\section{Results}

Resistance of wire and perforated film. The investigated nanostructures are shown in Fig. 1. One is a W-based nanowire of the width $w=50 \mathrm{~nm}$ made through focused-ion-beam-assisted deposition, and the other one is a 5-nm-thin disordered TiN film patterned into an array of holes with a diameter $\sim 120 \mathrm{~nm}$ and period $a=200 \mathrm{~nm}$. Both systems are extreme type-II superconductors in the dirty limit $\left(\ell<\xi_{\mathrm{d}}(0)\right.$, with $\ell$ being the mean free path and $\xi_{\mathrm{d}}(0)$ being the superconducting coherence length at zero temperature). In the wire, the superconducting critical temperature is $T_{\mathrm{c}}=4.3 \mathrm{~K}$ and $\xi_{\mathrm{d}}(0)=6.0 \mathrm{~nm}$. The as-grown continuous film has $T_{\mathrm{c}}=1.1 \mathrm{~K}$ and $\xi_{\mathrm{d}}(0)=9.3 \mathrm{~nm}$. Shown in Fig. $2 \mathrm{a}$ is the magnetic field dependence $R(B)$ of the resistance of the $\mathrm{W}$-based nanowire at different temperatures. At $T=4 \mathrm{~K}, R(B)$ dependence is monotonous. At lower temperatures, $R(B)$ develops a peculiar $\mathrm{N}$-shape that slightly shifts to higher fields when decreasing temperature. At lowest temperatures, $R(B)$ has a maximum and then drops into an extended reentrant zeroresistance region (within the noise level of $100 \mathrm{~m} \Omega$, high-field value is of $R_{\mathrm{N}}=3.56 \mathrm{k} \Omega$ ). The TiN film data are shown in Fig. $2 \mathrm{~b}$. Note that contrasting the wire behaviour, the film remains resistive even at $B=0$. This occurs because patterning drives the
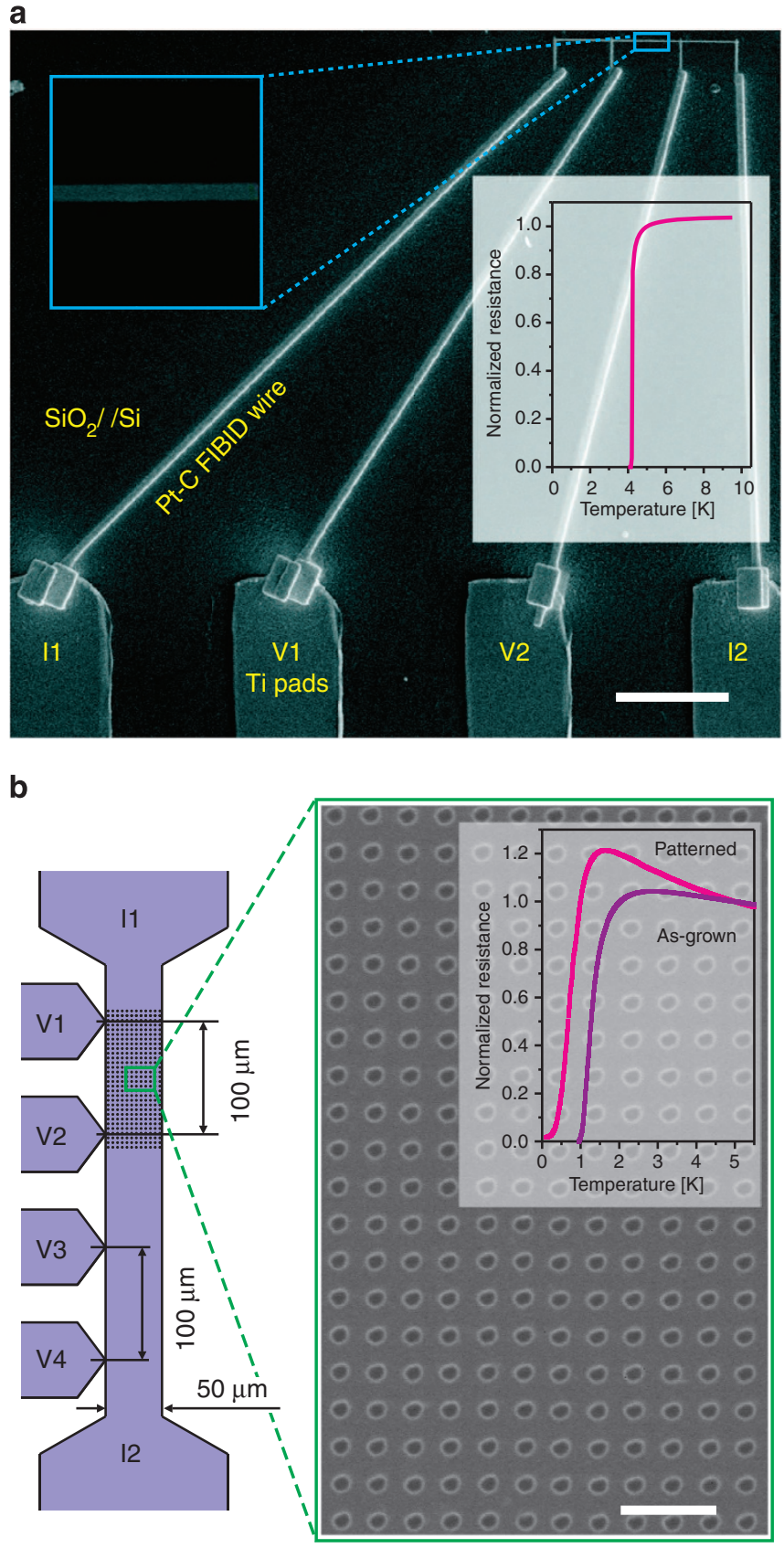

Figure 1 | Nanopatterned superconducting structures. (a) Scanning electron microscope (SEM) image of a W-based wire grown by FIBID technique on a $\mathrm{SiO}_{2} / \mathrm{Si}$ substrate. Main panel shows the experimental setup. The lateral size of the nanowire is $50 \mathrm{~nm}$. Contact pads are made of FIBID Pt-C wires. Scale bar, $5 \mu \mathrm{m}$. Left inset displays a zoom of the nanowire and the right inset shows the temperature dependence of the resistance in zero magnetic field normalized to the resistance at $T=5 \mathrm{~K}$, which is $R=3.56 \mathrm{k} \Omega$. (b) Left: a sketch of the measurement arrangement for the TiN-perforated film. The voltage probes $V 1-V 2$ fall within the perforated area, and the probes $V 3-V 4$ give a reference from the as-grown continuous film. Right: SEM image of the perforated film, consisting of an array of holes of $120 \mathrm{~nm}$ diameter with a period of $200 \mathrm{~nm}$. Scale bar, $500 \mathrm{~nm}$. The inset shows the temperature dependencies of the resistances of the thin film and the perforated film in zero magnetic field normalized to the resistance at $T=5 \mathrm{~K}$, which are $R=10.87 \mathrm{k} \Omega$ for as-grown film, and $R=64.93 \mathrm{k} \Omega$ for patterned film. Note that, in this case, the resistance does not drop to zero even at the lowest measured temperatures. See Methods for details, and Supplementary Fig. S1 for the current dependence, which remains ohmic at low bias. 
a

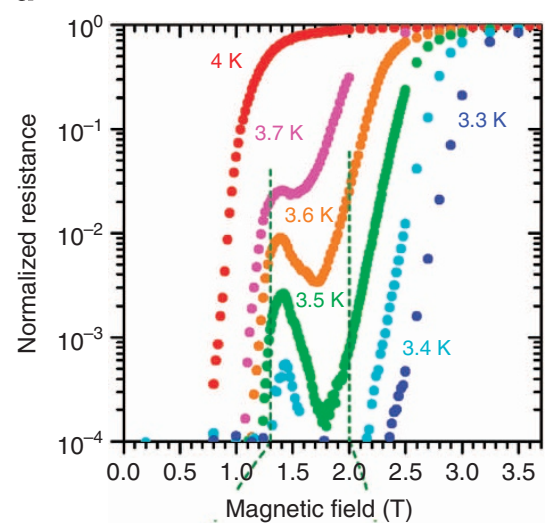

b

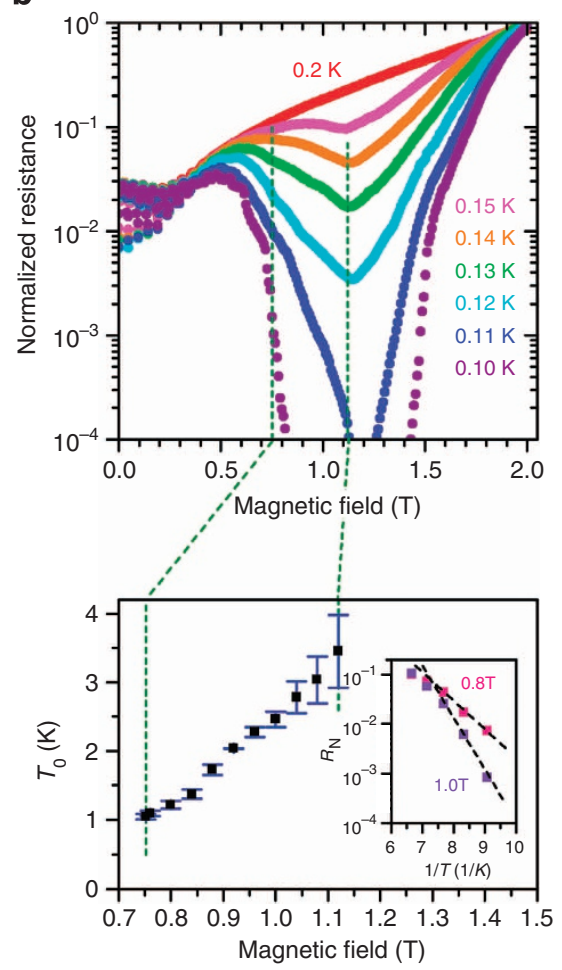

Figure 2 | Reentrance of the dissipation-free state. (a) The upper panel shows the magnetic field dependences of the resistance of the W-based nanowire taken at different temperatures, the data normalized to high-field values for respective temperatures. Note the large range of fields for reentrant superconductivity. Below $3.5 \mathrm{~K}$, the resistance becomes immeasurably small within nearly half a Tesla. The data at $T=3.3 \mathrm{~K}$ are normalized to the high-field resistance $R_{N}=3.56 \mathrm{k} \Omega$, the noise level is $100 \mathrm{~m} \Omega$, so the overall drop in $R(B)$ in the reentrant region is by the factor $10^{-4}$. The lower panel shows the variation of the activation energy $T_{0}$ with the magnetic field in the region of the formation of the reentrant dissipation-free state. Representative Arrhenius plots are given in the inset. (b) The upper panel presents the magnetic field dependences of the resistance of the perforated thin film normalized with respect to the value at $B=2 \mathrm{~T}$. At $B<0.5 \mathrm{~T}$, the magnetoresistance shows oscillations with the period $\Delta B=\pi \hbar /\left(e a^{2}\right)$, where $a$ is the pattern unit size. These oscillations are characteristic to weak-link wire networks and are described in Supplementary Fig. S2. At and below $T=0.15 \mathrm{~K}$, the curves develop pronounced $\mathrm{V}$-shape with the position of the minimum almost independent of the temperature. Reentrant superconductivity appears at $T=0.11 \mathrm{~K}$, and extends over a field interval of nearly $0.7 \mathrm{~T}$ at $T=0.1 \mathrm{~K}$. In the region $0.9 / 1.35 \mathrm{~T}$, the resistance is below the noise level of $2 \mathrm{~m} \Omega$. The normalized resistance at $2 \mathrm{~T}$ is about $200 \mathrm{k} \Omega$, so that at $T=0.1 \mathrm{~K}$, the resistance drops by factor $10^{-7}$. We show only four orders of magnitude for $R(B)$ in a logarithmic scale to match the data on a wire. The lower panel shows the growth of the activation energy in the field range corresponding to the ascending branch of the magnetoresistance. The inset demonstrates the representative Arrhenius plots. Error bars in the main bottom panels of $\mathbf{a}$ and $\mathbf{b}$ represent standard deviations from the linear fit shown in the insets as dashed lines.

Berezinskii-Kosterlitz-Thouless transition temperature $T_{\mathrm{BKT}}$ of the film down to temperatures lower than those of present experiment ${ }^{12}$, and thus unbound vortices and antivortices move freely under the applied current leading to finite resistance. Upon increasing magnetic field, $R(B)$ of the film develops a dip around $0.4 \mathrm{~T}$, which again becomes very pronounced at lowest temperatures. At $T=0.10 \mathrm{~K}$, the resistance vanishes into the noise over a field range of about $0.7 \mathrm{~T}$ (in the interval $0.9 / 1.35 \mathrm{~T}$ and at $T=0.1 \mathrm{~K}$, noise level is $R=2 \mathrm{~m} \Omega$ and high-field resistance $R(B=5 \mathrm{~T})=200 \mathrm{k} \Omega$, meaning a drop over seven orders of magnitude). The decrease in the resistance is roughly exponential as function of temperature in both systems $R \propto \exp \left[T_{0}(B) / T\right]$. The corresponding activation energy $T_{0}(B)$ is shown in the lower panels of Fig. 2, and increases by a factor of three with increasing magnetic field.

Theoretical model: phase diagram. To get insight into the mechanism of the observed non-monotonic $R(B)$ behaviour and reentrant zero-resistance state, we review the thermodynamics of a superconducting wire (strip) of the width $w \gtrsim \xi_{\mathrm{d}}$. Following the calculations presented in ref. 13 for the slab with the width $w \gtrsim \xi_{\mathrm{d}}$ in a parallel field, we deduce the equilibrium phase diagram shown in Fig. 3a. At fields $B<B_{\mathrm{V}}$, the superconducting and normal states are separated by the thermodynamic critical field $B_{\mathrm{c}}=\sqrt{3} \Phi_{0} /\left(\pi \xi_{\mathrm{d}} w\right)$, with $\Phi_{0}=\pi \hbar c / e$ being the flux quantum ${ }^{14}$. At high fields, $B \gg B_{\mathrm{V}}$, superconductivity vanishes at the upper critical field for surface superconductivity $B_{\mathrm{c} 3}=1.695 B_{\mathrm{c} 2}$, where $B_{\mathrm{c} 2}=\Phi_{0} / 2 \pi \xi_{\mathrm{d}}^{2}(T)$. The field $B_{\mathrm{V}}$ divides bulk and surface superconductivity regions: At $B<B_{\mathrm{V}}=1.61 \dot{\mathrm{s}}\left(\Phi_{0} / w^{2}\right)$, the amplitude of the superconducting order parameter is maximal at the middle line of the wire and is suppressed towards the edges, while at $B>B_{\mathrm{V}}$, the situation is reverse and superconductivity subsists at the surface (edge) sheath ${ }^{13,15}$. Equilibrium vortices settle in a strip at $B=B_{\mathrm{V}}$ as a one-dimensional vortex row along the median ${ }^{13}$. Within the parameter range of our experiment, the wire accommodates only one vortex row. The point $\left\{T_{\mathrm{V}}, B_{\mathrm{V}}\right\}$ where $B_{\mathrm{V}}=B_{\mathrm{c}}(T)$ is a tri-critical point. We find in our wire, $B_{\mathrm{V}}=1.3 \mathrm{~T}$ and $T_{\mathrm{V}}=3.87 \mathrm{~K}$. These values coincide with very good precision with the experimentally found magnetic field of the maximum in $R(T) \quad\left(B_{\max } \approx 1.4 \mathrm{~T}\right)$ and the experimental temperature above which no maximum is observed $(\approx 3.9 \mathrm{~K})$. Numerical computations of Ginzburg-Landau equations (Supplementary Discussion and ref. 16) illustrate suppression of 
a

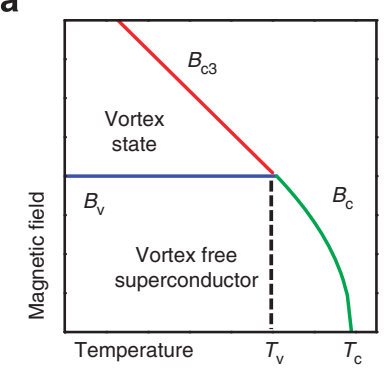

C

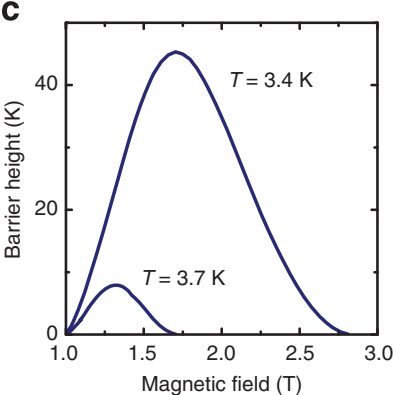

b
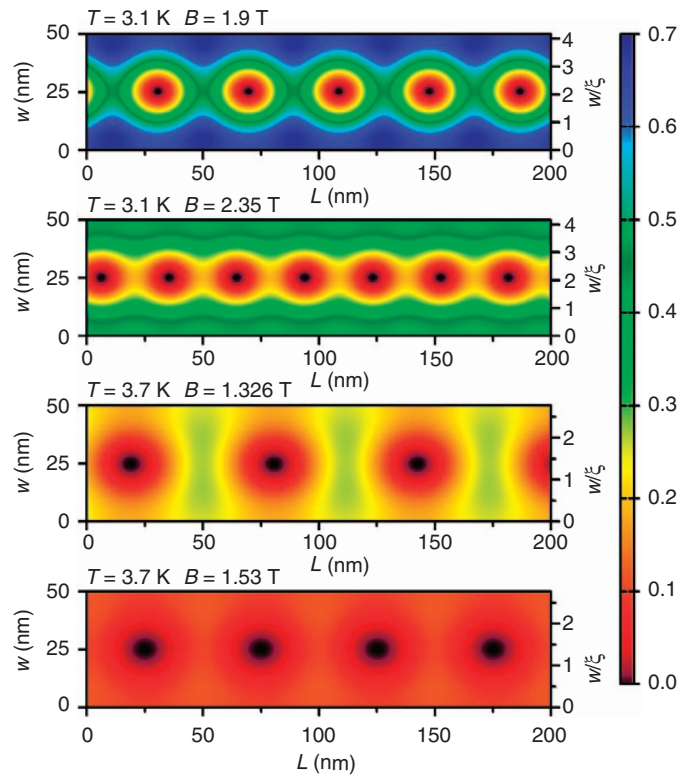

Figure 3 | Theory for the superconducting wire. (a) Sketch of the phase diagram obtained within the framework of the Ginzburg-Landau description of the superconducting wire with $w \gtrsim \xi$ (ref. 13). The thermodynamic magnetic field $B_{c}$ separates the vortex-free state from the normal metal at $B<B_{\vee}$, at $B>B_{V}$ the upper-surface critical field $B_{c 3}$ has this role. When $B_{c}=B_{V}$, we find a tri-critical point in the phase diagram, whose position coincides with the temperature $T_{V}$ above which vortices do not enter the wire, because the coherence length $\xi_{\mathrm{d}}$ has grown over the lateral size of the wire. Calculations give $B_{V}=1.4 \mathrm{~T}$ and $T_{V}=3.9 \mathrm{~K}$ for the wire parameters (Fig. 1). (b) Evolution of the order parameter in the superconducting wire with the applied magnetic field. The colour map of the square modulus of the order parameter $|\Psi|^{2}$ for representative temperatures and magnetic fields from numerical solution of the Ginzburg-Landau equations. Colour scale is the same for all four panels. See Supplementary Discussion for details. We use a wire with $w=50 \mathrm{~nm}$ and material parameters as in the experiment. The upper two panels show the increase in linear vortex density above $B_{\mathrm{V}}$. Temperature is too low for vortex motion in the resistance. The two lower panels show, respectively, the order parameter distribution at the magnetic field where barrier height is maximal, that is, close to the minimum of resistance, and close to $B_{\mathrm{c} 3}$. (c) The activation barrier for single vortex motion as a function of the magnetic field for $T=3.4$ and 3.7 K. Non-monotonic behaviour of the barriers reflects the competition between reduced vortex motion, and destruction of superconductivity. The obtained barrier energy is roughly of the same order as the energies discussed in Figs 2 and 4 .

superconducting order parameter near the middle line and onset of surface-like superconductivity at $B>B_{\mathrm{V}}$ (Fig. 3b). The temperature above which no vortices appear in the wire $(3.8 \mathrm{~K})$ is also in a good agreement with $T_{\mathrm{V}}$, which is also close to temperature defining the condition ${ }^{17} w>1.85 \xi_{\mathrm{d}}(T)$ for vortices to first enter the wire.

Theoretical model: calculation of the resistance. With the phase diagram at hands, we are now equipped to explain the magnetic field dependence of the resistance $R(B)$. At $B<B_{\mathrm{V}}$, the dissipation is caused by the 'external' vortices crossing the wire. Every vortex sees a dome-like potential barrier with the maximum at the strip median (Supplementary Discussion and ref. 18) and the height linearly decreasing with $B$. Correspondingly, $R \propto \exp \left(B / B^{*}\right)$ with $B^{\star}=0.028 \mathrm{~T}$ at $T=4 \mathrm{~K}$ in a fair agreement with the experimental data.

At $B=B_{\mathrm{V}}$, a single row of equilibrium vortices settles in the middle of the wire. Thus, at $B>B_{\mathrm{V}}$ the dissipation is governed by the escape of these vortices from the strip across the barriers created by edge superconductivity. At $B \approx B_{\mathrm{V}}$, the newly established edge barriers are nearly zero. As they also vanish at $B=B_{\mathrm{c} 3}$, where superconductivity is fully suppressed, these barriers must achieve their maximal value at some field between $B_{\mathrm{V}}$ and $B_{\mathrm{c} 3}$ giving rise to the N-shape of $R(B)$ with the maximum at $B_{\mathrm{V}}$. To quantify suppression of $R(B)$, we note that as the edge superconducting sheath at $B>B_{\mathrm{V}}$ has a width of $\sim \xi_{\mathrm{d}}$, a vortex crossing the edge barrier can be viewed as a phase slip (PS) in the sheath ${ }^{19,20}$. To derive the PS activation energy $E_{\mathrm{PS}}$, we employ the concept of the effective resistive medium ${ }^{21}$. There the median band containing vortices can be considered a normal metal with the effective density of normal excitations $v(B)=$ $\left(B / B_{\mathrm{c} 3}\right) v_{\mathrm{N}}$, namely the density of excitations within a single core $\left(v_{\mathrm{N}}\right)$ times the dimensionless linear vortex density $\left(B / B_{\mathrm{c} 3}\right)$. Then the activation energy governing the resistance is the PS nucleation energy, $E_{\mathrm{PS}} \simeq E_{\mathrm{C}} \mathcal{F}(B)$, where $E_{\mathrm{C}}=2 v_{\mathrm{N}} \Delta^{2} \xi_{\mathrm{d}}^{2} s$ is the condensation energy at zero field, $\Delta$ is the superconducting gap and $\xi_{\mathrm{d}}^{2} s$ is the volume of the PS nucleus $\left(s=\min \left\{\xi_{\mathrm{d}}(T), d\right\}\right.$ and $d$ being the thickness of the sample (Supplementary Discussion), and $\mathcal{F}(B)$ is a dimensionless function of the magnetic field such that $\mathcal{F}(B) \sim B / B_{c 3}$ at $B \ll B_{\mathrm{c} 3}$, and $\mathcal{F}(B) \approx\left(1-B / B_{c 3}\right)^{5 / 4}$ at $B \rightarrow B_{\mathrm{c} 3}$ (Supplementary Discussion and Supplementary Table S1)). As $\mathcal{F}(B)$ varies from $\approx 0.15$ to 0.4 and $E_{C}(T) \approx 25 k_{\mathrm{B}} T_{\mathrm{c}}$ for the strip, the resistance $R(B) \propto c \exp \left(-E_{\mathrm{PS}} / T\right)$ is indeed effectively suppressed over a large field interval between $B_{\mathrm{V}}$ and $B_{\mathrm{c} 3}$ at $T<3.5 \mathrm{~K}$. The remarkable effect of the increase of $E_{\mathrm{PS}}$ with the magnetic field reflects the fact that at $B \gtrsim B_{\mathrm{V}}$, the 'metallicity' of the resistive central layer of the wire (that is, the effective density of the normal excitations near the median) increases faster than the suppression of surface superconductivity at the edges. The field-induced metallicity means merely an incremental suppression of the order parameter along the median by an increasingly dense vortex chain. Therefore, densely packed vortices dig the more and more deep potential well for themselves with increasing magnetic field and arrest their transverse motion. The current is short circuited by the edge 
superconducting channels and the wire turns superconducting. Upon further increase of magnetic field, the suppression of edge superconductivity takes over and $R(B)$ starts to grow again.

That the escape barriers for a vortex chain in the narrow superconducting slab can grow with the magnetic field and lead to enhancement of superconducting parameters was discussed in ref. 22. Unfortunately, while correctly capturing the trend, the image approach used in ref. 22 did not take into account the collective suppression of the order parameter near the median and thus underestimated the magnitude of the escape barrier. As a result, the effect came out significantly reduced. More precise Ginzburg-Landau simulations accounting for the self-induced suppression of the order parameter along the vortex chain show gigantic increase of the escape barrier with the magnetic field agreeing with the effective medium-based estimates (Fig. 3b,c).

Turning to the film, we find the same mechanism of fieldpromoted metallicity responsible for the reentrant dissipationfree state. As we have already mentioned, the zero-field background resistance results from the free motion of vortices and antivortices, because $T_{\mathrm{BKT}}$ (below which they get bound into neutral pairs) is lower than the temperature of our measurements ${ }^{12}$. At $B \lesssim 0.5 \mathrm{~T}$, the additional vortices induced by the field are trapped in the holes. Accordingly, the order parameter is suppressed around the holes and is maximal within the diamond-like areas between the holes. The perforated film behaves as a weak-link network and the resistance exhibits oscillations on top of the background resistance as a function of the magnetic field ${ }^{12}$ (Supplementary Fig. S1), with the period corresponding to integer number of flux quanta per hole. This, so far, is similar to usual 'vortex-matching effect,' as seen in the arrays of holes in superconducting films and weak link networks (for example, refs 23, 24). When the amount of vortices in each hole is so large that the currents around holes reach the critical current magnitude, vortices cannot be trapped within holes any more and start to fill the diamond-like areas between them. From this moment, the coherent effects characteristic to weak-link network are suppressed, as evidenced by the disappearance of oscillations in $R(B)$. The behaviour of superconductivity surrounding the holes inverts. Vortices piling up in diamondlike cells between the holes suppress the order parameter, which becomes maximal in the rings of width $\xi_{\mathrm{d}}$ surrounding the holes. These superconducting rings induce superconductivity in the isthmuses between the holes via the proximity effect and bridge each other to merge into a chain-mail-like network of linked superconducting channels of width $\simeq \xi_{\text {d }}$ across the film (Fig. 4). The superconducting chain-mail short-circuits the supercurrent across the film similar to edge channels in a wire. The hopping of fluxons between the holes is blocked, as the holes are fully filled up. Vortices clustering in the diamond-like inter-hole cells suppress the nearby order parameter and self arrest themselves. We can understand vortex motion across the superconducting chain mail, which partitions each cell, as phase slips through 1D channels. Each vortex-containing cell can again be described as a resistive effective medium with the effective density of states of normal excitations $v(B) \simeq\left(B / B_{\mathrm{c} 3}\right) v_{\mathrm{N}}$. Note that the diameter of the holes is twelve times $\xi_{\mathrm{d}}$. Thus, as the size of the PS nucleus along the channels is about $\xi_{\mathrm{d}}$, the curvature of chain-mail elements does not change noticeably the PS nucleus energy $E_{\mathrm{PS}}$. One thus expects that the geometric form-factor $\mathcal{F}(B)$, describing the competition between the increasing field-induced metallicity of the resistive parts and the field suppression of surface barriers posed by superconducting channels, is nearly the same for the strip and the film.

To establish that the mechanism of reentrant superconductivity is unique, we plot in Fig. 4 the measured activation barriers at the descending branches of $R(B)$ for both the nanowire and the film,

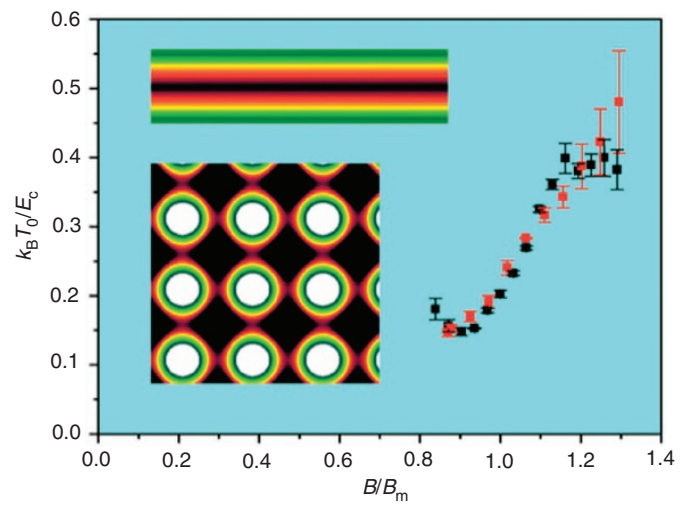

Figure 4 | Phase slip activation barrier. The experimentally measured activation energy for the descending branch of the $R(B)$ for the superconducting wire (black symbols) and for the perforated thin film (red symbols) in units of $E_{O}$ (Fig. 2) as a function of the magnetic field normalized to the centre of the magnetic field region where the resistance decreases (between vertical dashed lines in Fig. 2). Error bars represent standard deviations from the fit described in the text in relation to Fig. 2. The upper panel of the inset shows the nearly tri-layered state leading to recovered superconductivity in a wire. The inner (black) stripe is a nearly metallic state and the green layers show the edge-enhanced superconductivity. The lower inset panel presents corresponding superconducting state in a perforated film. The superconducting annuli surrounding the holes induce superconductivity in the interhole constrictions and results in a chain-mail-like configuration.

normalized to their respective nucleus energies $E_{C}$ (reflecting specific properties of each material), as a function of the normalized magnetic field $B / B_{\text {min }}$. For the nanowire $E_{\mathrm{C}} / k_{\mathrm{B}}=101.3 \mathrm{~K}$ at $T=3.2 \mathrm{~K}$, and $E_{\mathrm{C}} / k_{\mathrm{B}}=7.18 \mathrm{~K}$ for the film at $T=0.12 \mathrm{~K}$. Both curves collapse on top of each other evidencing the universal character of the $F(B)$ function and thus, the common mechanism for the reentrance of the dissipation-free state in a strip and a patterned film.

\section{Discussion}

We have demonstrated giant magnetic field-induced reentrant superconductivity in two seemingly disparate systems, a thin W-based wire and thin TiN film patterned into an array of holes. By juxtaposing their respective $R(B)$ behaviours and system parameters, we have revealed the underlying mechanism of the effect. The giant reentrance rests on two components. The first is that surface (edge) superconductivity arrests vortex motion by constricting them into immobile aggregates: the denser the vortex cluster, the better the superconducting order parameter around this cluster is suppressed and the deeper the self-generated potential well confining vortices. The effective resistive medium grows more metallic with field. The density of normal excitation states is proportional to $B$, and so does the energy of the PS nucleus. Thus, increasing magnetic field suppresses the phase slips across the edge superconducting channels until the suppression of surface superconductivity itself prevails. The second component is the specific geometry ensuring that the volume occupied by edge superconducting channels is comparable to that of the vortex-carrying resistive medium. In that case, collective depinning effects do not develop, implying that the surface barrier for vortex escape can stand applied currents comparable to the pair-breaking current. Our findings open new routes to design superconducting devices working at high magnetic fields and temperatures, and mark an important step towards resolving the long-standing problem of extending the 
coherent dissipation-free superconducting state to high field and temperature range.

\section{Methods \\ Fabrication and characterization of the wire. Amorphous $\mathrm{W}$-based super-} conducting nanowires were grown by the focused ion beam-induced deposition (FIBID) technique in a dual beam, Nova 200 Nanolab (FEI Company) equipment. FIBID consists in the local growth of nanostructures on a substrate where the suitable precursor gas molecules are adsorbed and dissociated by the focused ion beam (FIB $)^{25}$. The technique can be viewed as a local chemical vapour deposition method induced by the FIB. The typical lateral size and the thickness obtained using the FIBID range from few nanometres to several micrometres, and, under appropriate conditions described below, the superconducting nanostructures can be obtained. As FIBID is a direct-write technique, it does not involve the succession of complex procedures needed to make other superconducting nanostructures, such as electrochemical deposition or carbon nanotube templates, which have been used in the past to create small superconducting wires ${ }^{26}$

We were using the Ga-based FIB in combination with the precursor gas $\mathrm{W}(\mathrm{CO})_{6}$. It has been shown that the resulting deposit is superconducting with $T_{\mathrm{c}}$ around $5 \mathrm{~K}$ (ref. 27). The precursor gas was injected into the process chamber by means of a needle inserted in the vicinity of the specimen, around $50 \mu \mathrm{m}$ away in $x-y$ direction and around $150 \mu \mathrm{m}$ away in the $z$ direction of the substrate surface. This allows for an efficient FIBID process. A silicon wafer with the 250-nm thermally grown oxide layer was used as a substrate. The nanowire was grown in the geometry shown in Fig. 1, having four pads made of the same material as the nanowire itself. At the first stage, a horizontal nanowire of the $8-\mu \mathrm{m}$ length and four perpendicular equidistant nanowires of the $2-\mu \mathrm{m}$ length, used for the I1, V1, $\mathrm{V} 2$ and $\mathrm{I} 2$ connexions, were deposited. The used FIBID parameters were as follows: the ion beam acceleration voltage was $30 \mathrm{kV}$; the ion beam current was $1 \mathrm{pA}$; the dwell time was $200 \mathrm{~ns}$; the scanning pitch was $10.5 \mathrm{~nm}$; the chamber base pressure was $1 \times 10^{-6} \mathrm{mbar}$; the chamber pressure during FIBID was $6 \times 10^{-6} \mathrm{mbar}$; and the precursor temperature was $55^{\circ} \mathrm{C}$. The in situ scanning electron microscope images shown in Fig. 1 were performed by the same equipment using a fieldemission electron gun, thus providing high-resolution images of the nanowires and giving a good determination of the lateral size $(50 \mathrm{~nm})$. Additionally, we made atomic force microscopy measurements, giving a good thickness uniformity $(30 \mathrm{~nm}$ with a maximum variation of $\pm 5 \mathrm{~nm}$ ) along the length of nanowires.

The set-up for performing measurements on the nanowire is shown in Fig. 1. The Pt-C wire was grown by FIBID on the top of the four small paths of the nanowire (using $\left(\mathrm{CH}_{3}\right)_{3} \mathrm{Pt}\left(\mathrm{CpCH}_{3}\right)$ as the precursor gas), connecting the nanowire to $\mathrm{Ti}$ pads where aluminium wires could be bonded. The Ti pads were made using the optical lithography lift-off method and e-beam evaporation with a thickness of $150 \mathrm{~nm}$. More details of the contacting method are given in refs $28,29$.

Magnetotransport measurements were performed using a commercial Physical Properties Measurements System (PPMS from Quantum Design) in the temperature range from $300 \mathrm{~K}$ down to $2 \mathrm{~K}$. The magnetic field was applied perpendicular to the substrate and the ac measurement mode was used. The composition of the $\mathrm{W}$-based FIBID nanostructures in atomic percentages are as follows. The $\mathrm{W}$ percentage: $40 \pm 7 \%$; C percentage: $43 \pm 4 \%$, Ga percentage: $10 \pm 3 \%$, O percentage: $7 \pm 2 \%$. The composition remains the same through the full thickness ${ }^{30}$.

Superconducting properties of the W-based FIBID thin films of equal composition and the same growth methods have been previously studied in refs 30,31 . The classical vortex lattice behaviour was found, with $B_{\mathrm{c} 2}(T)$ following a clear mean field behaviour ${ }^{30}$. The Ginzburg-Landau parameters were calculated from the normal resistance of $2.75 \mu \Omega \mathrm{m}$ and the derivative of upper critical field at $T_{\mathrm{c}}$, namely $\left(\mathrm{d} B_{\mathrm{c} 2} / \mathrm{d} T\right)\left(T=T_{\mathrm{c}}\right)=2 \mathrm{TK}^{-1}$, resulting in $\xi(0)=6 \mathrm{~nm}, \lambda(0)=640 \mathrm{~nm}$, and the GL parameter $\kappa=\lambda(0) / 1.63 \xi(0)=65$. The thermodynamic critical field is $B_{\mathrm{c}}=40 \mathrm{mT}$, the upper critical magnetic field $B_{\mathrm{c} 2}=6.5 \mathrm{~T}$ and the superconducting gap is $\Delta_{0}=0.66 \mathrm{meV}$. A neat Abrikosov hexagonal vortex lattice was observed in the films through scanning tunneling microscopy and spectroscopy (STM/S) ${ }^{30,31}$. The material parameters inferred from the data are as follows: the diffusion coefficient $D=0.51 \mathrm{~cm} \mathrm{~s}^{-1}, R_{\square}=59.3 \Omega$, and the density of normal excitation states $v=\sigma /\left(e^{2} D\right) \equiv 1 /\left(e^{2} D R_{\square} d\right)=4.3 \times 10^{47} \mathrm{~J}^{-1} \mathrm{~m}^{-3}$.

Fabrication and characterization of the perforated film. A smooth, continuous and uniform TiN film with the thickness of $5 \mathrm{~nm}$ was synthesized by atomic layer deposition on a $\mathrm{SiO}_{2} / \mathrm{Si}$ substrate at the deposition temperature $T_{\mathrm{d}}=350{ }^{\circ} \mathrm{C}$. Highresolution transmission electron microscopy was performed using a JEOL-4000EX (Japan) microscope operated at $400 \mathrm{keV}$. Electron transmission micrographs and diffraction patterns revealed the polycrystalline structure with the densely packed crystallites (Supplementary Fig. S2) (the average grain size is about $5 \mathrm{~nm}$ ). The cross-section micrograph of the film (Supplementary Fig. S2c) shows the atomically smooth interface between the $\mathrm{SiO}_{2}$ substrate and the TiN film and the atomically smooth surface of the TiN film.

To carry out the transport measurements the film was first patterned by means of the conventional UV lithography and plasma etching into the bridges $50 \mu \mathrm{m}$ wide and with the $100 \mu \mathrm{m}$ distance between the voltage probes (Fig. 1b). Then, making use of the electron lithography and the subsequent plasma etching, a square lattice of holes with the diameter $\sim 120 \mathrm{~nm}$ and the period $a=200 \mathrm{~nm}$ covering the $50 \times 120 \mu^{2}$ area, was created (Fig. 1b). The voltage probes V1-V2 were designed to fall within the nanopatterned domain of the film, while the probes V3-V4 were placed within its continuous (non-patterned) section to measure thus the resistance of the original continuous film. The nanopatterned section of the film confined between the probes V1-V2 contained $50 \mu \mathrm{m} \times 100 \mu \mathrm{m} /$ $(200 \mathrm{~nm})^{2}=125,000$ elemental units. As a first step of the electron lithography process, the films were spin-coated with the poly(methyl methacrylate) (PMMA) electron-beam resist with the molecular weight 950.000 diluted with anisole, providing the thickness of $180 \mathrm{~nm}$ after $30 \mathrm{~s}$ spinning at 3,000 rpm. The samples coated with PMMA were subsequently baked over $30 \mathrm{~min}$ in convection oven at the temperature $170^{\circ} \mathrm{C}$. The desired network pattern was created by the exposure of the PMMA layer to the electron beam using Raith 150 high-resolution electronbeam lithography system (the diameter of the beam is of about $2.4 \mathrm{~nm}$ at $20 \mathrm{kV}$ ). The PMMA was used as a positive electron-beam resist, as it provides high contrast with respect to further developing. Upon removing of the exposed parts of the PMMA by the standard methyl-isobutyl-ketone (MIBK)-isopropyl 1:3 solution, the pattern mask have got formed. Usually in the electron-beam lithography systems the dots are designed as an array of single elements with the quantity equal to the quantity of dots. Then the time necessary for settling the unique element gets critical for the overall time of the structure exposure. To design our TiN-nanopatterned samples, we have developed a method, which allows the exposure of the array of $10^{9}$ dots over the time as short as a few tenths of minutes. The strategy of method was to write a large rectangle, using a step equal to the array step. The size of dots depended on the area dose and could be varied from $20 \mathrm{~nm}$ up to $120 \mathrm{~nm}$ as a result of the Gaussian shape of electron beam and of the proximity effect. We have determined that the best patterning results are achieved if the energies of the electrons in the beam exceed or are equal to $20 \mathrm{kV}$, and the apertures size are small enough, about $7.5 \mu \mathrm{m}$, to ensure the round shape of dots. The chosen design (pairs of V1-V2 and V3-V4 probes) allows comparing the physical properties of the patterned sections, the non-patterned sections of the film that were subject to whole procedure except for being exposed to electron beam and the films that were not subject to the electron-beam lithography process at all. The superconducting and transport properties of the second and the latter appeared the same, proving thus that the electron-beam lithography treatment did not damage the TiN films and remained identical to the films that were fully characterized by the infrared ${ }^{32}$ sample D15 and low-temperature scanning tunnelling spectroscopy ${ }^{33,34}$, sample TiN2.

The temperature $T$ and the magnetic field $B$ dependences of the resistance were measured using the standard four-probe low-frequency ac techniques at the frequency $1 \mathrm{~Hz}$ with an ac current $0.3 \mathrm{nA}$. So the current was sufficiently small to ensure the linear response regime as was verified by direct measurements of the current-voltage $(I-V)$ characteristics (Supplementay Fig. S1b). The magnetic field was applied perpendicular to the film surface. Transport and superconducting properties of our sample are close to those used in earlier studies ${ }^{12,35}$. The sample parameters are as follows: the diffusion constant $D=0.32 \mathrm{~cm}^{2} \mathrm{~s}^{-1}$, the superconducting coherence length $\xi(0)=9.3 \mathrm{~nm}$, the transition temperature, $T_{\mathrm{c}}=1.115 \mathrm{~K}, \lambda(0)=2.4 \mu \mathrm{m}, \kappa=158$ and the superconducting gap $\Delta=0.22 \mathrm{meV}$. Further, using the TiN film room temperature resistance per square $R_{\square}=$ $\left(e^{2} D v d\right)^{-1}=2.94 \mathrm{k} \Omega$, we estimate the density of states as $v=8.3 \times 10^{46} \mathrm{~J}^{-1} \mathrm{~m}^{-3}$

Shown in the Supplementary Fig. S1 are the data on the transport measurements of the TiN-based perforated film. Supplementary Figure S1a displays oscillations of the magnetoresistance observed in the range $B<0.5 \mathrm{~T}$ up to temperatures $\gtrsim 0.7 \mathrm{~K}$ evidencing the existence of the superconducting order parameter in the temperature range in question. These oscillations are characteristic to $2 \mathrm{D}$ Josephson junction arrays, proximity-effect junction arrays and superconducting wire networks, and reflect the collective behaviour and effect of synchronization of the Josephson phases in 2D multiconnected superconducting systems, for detailed discussion see ref. 35 . Supplementary Figure S1b shows the $I-V$ characteristic in a $\log -\log$ scale and the inset presents the same data as the differential resistance $\mathrm{d} V /$ $\mathrm{d} I$ versus $I$ plot at zero magnetic field and $T=0.1 \mathrm{~K}$. Using the magnitude of the critical current evaluated from the position of the maximum of the $\mathrm{d} V / \mathrm{d} I$ versus curve $^{35}$, we have determined the critical current through the whole network $I_{\mathrm{c}}=0.17 \mu \mathrm{A}$, yielding the critical current $i_{\mathrm{c}}=0.68 \mathrm{nA}$ per one constriction. Having determined the critical current, one estimates the Pearl penetration depth $\lambda_{\perp}=\hbar$ / $\left(2 e \mu_{0} i_{\mathrm{c}}\right)=38 \mathrm{~cm}$, and the Josephson coupling $E_{\mathrm{j}}=\left(\hbar i_{\mathrm{c}} / 2 e\right)$. It yields $E_{\mathrm{j}} / k_{\mathrm{B}} \simeq 0.016 \mathrm{~K}$, and, accordingly, $T_{\mathrm{BKT}}=\pi E_{\mathrm{J}} /\left(2 k_{\mathrm{B}}\right) \simeq 0.025 \mathrm{~K}$.

\section{References}

1. Schubnikow, L. W., Chotkewitsch, W. I., Schepelew, J. D. \& Rjabinin, J. N. Magnetische Eigenschaften supraleitender Metalle und Legierungen. Phys. Z. Sowiet. 10, 165-192 (1936).

2. Schubnikov, L. W., Khotkewitsch, V. I., Schepelev, Yu. D. \& Rjabinin, Yu. N Magnetic properties of superconducting metals and alloys. Zh. Eksp. Teor. Fiz 7, 221-237 (1937)

3. Abrikosov, A. A. On the magnetic properties of second kind superconductors Sov. Phys. JETP 5, 1174-1182 (1957).

4. Anderson, P. W. \& Kim, Y. B. Hard superconductivity-theory of motion of Abrikosov flux lines. Rev. Mod. Phys. 36, 39-43 (1964). 
5. Campbell, A. M. \& Ivetts, J. E. Critical Currents in Superconductors Monographs on Physics (Taylor \& Francis Ltd., 1972).

6. Larbalestier, D., Gurevich, A., Feldmann, D. M. \& Polyanskii, A. High- $T_{c}$ superconducting materials for electric power applications. Nature 414, 368-377 (2001).

7. Blatter, G., Feigel'man, M., Geshkenbein, V., Larkin, A. \& Vinokur, V. Vortices in high temperature superconductors. Rev. Mod. Phys. 66, 1125-1388 (1994).

8. Parks, R. D. \& Mochel, J. M. Evidence for quantized vortices in a superconducting strip. Phys. Rev. Lett. 11, 354-358 (1963).

9. Hlásnik, I. et al. Properties of superconducting NbTi superfine filament composites with diameters $\lesssim 0.1 \mu \mathrm{m}$. Cryogenics 25, 558-565 (1985).

10. Xiong, P., Herzog, A. \& Dynes, R. C. Negative magnetoresistance in homogeneous amorphous superconducting $\mathrm{Pb}$ wires. Phys. Rev. Lett. 78, 927-930 (1997)

11. Rogachev, A. et al. Magnetic-field enhancement of superconductivity in ultranarrow wires. Phys. Rev. Lett. 97, 137001 (2006).

12. Baturina, T. I. et al. Nanopattern-stimulated superconductor-insulator transition in thin TiN films. Europhys. Lett. 93, 47002 (2011).

13. Saint-James, D., Thomas, E. \& Sarmas, G. Type II Superconductivity (Pergamon, 1969)

14. Ginzburg, V. On the behavior of superconducting films in a magnetic field. Dokl. Akad. Nauk SSSR 83, 385 (1952).

15. Saint-James, D. \& de Gennes, P. Onset of superconductivity in decreasing fields. Phys. Lett. 7, 306-308 (1963).

16. Palacios, J. Vortex lattices in strong type-II superconducting two-dimensional strips. Phys. Rev. B 57, 10873-10876 (1998).

17. Fink, H. Vortex nucleation in a superconducting slab near a second-order phase transition and excited states of the sheath near $H_{\mathrm{c} 3}$. Phys. Rev. B 177, 732-737 (1969).

18. Kuit, K. et al. Vortex trapping and expulsion in thin-film $\mathrm{YBa}_{2} \mathrm{Cu}_{3} \mathrm{O}_{7 \delta}$ strips Phys. Rev. B 77, 134504 (2008).

19. Aranson, I. \& Vinokur, V. Surface instabilities and vortex transport in currentcarrying superconductors. Phys. Rev. B 57, 3073-3083 (1998).

20. Anderson, P. Consideration on the flow of superfluid helium. Rev. Mod. Phys. 38, 298-310 (1966)

21. Abrikosov, A. Fundamentals of the Theory of Metals (Elsevier Science Pub. Co, 1988).

22. Takács, S. Properties of superfine superconducting filaments embedded in normal matrix. Czech. J. Phys. B 36, 524-536 (1986).

23. Baert, M., Metlushko, V., Jonckheere, R., Moshchalkov, V. \& Bruynseraede, Y. Composite flux-line lattices stabilized in superconducting films by a regular array of artificial defects. Phys. Rev. Lett. 74, 3269-3272 (1995).

24. Martin, J., Velez, M., Hoffmann, A., Schuller, I. \& Vicent, J. Temperature dependence and mechanisms of vortex pinning by periodic arrays of $\mathrm{Ni}$ dots in Nb films. Phys. Rev. B 62, 9110-9116 (2000).

25. Utke, P., Hoffmann, J. \& Malngailis, J. Gas-assisted focused electron beam and ion beam processing and fabrication. J. Vac. Sci. Technol. B 26, 1197-1277 (2008).

26. Bezryadin, A., Lau, N. \& Tinkham, M. Quantum suppression of superconductivity in ultrathin nanowires. Nature 404, 971-974 (2000)

27. Sadki, E., Ooi, S. \& Hirata, K. Focused-ion-beam-induced deposition of superconducting nanowires. Appl. Phys. Lett. 85, 6206-6209 (2008).

28. De Teresa, J. M., Córdoba, R., Fernández-Pacheco, A., Montero, P., Strichovanec, R. \& Ibarra, M. R. Origin of the difference in the resistivity of as-grown focused-ion- and focused-electron-beam-induced $\mathrm{Pt}$ nanodeposits. J. Nanomat. 936863 (2009).
29. Fernández-Pacheco, A., De Teresa, J. M., Córdoba, R. \& Ibarra, M. R. Metalinsulator transition in Pt-C nanowires grown by focused-ion-beam-induced deposition. Phys. Rev. B 79, 174204 (2009).

30. Guillamón, I. et al. Nanoscale superconducting properties of amorphous W-based deposits grown using a focused-ion-beam. New J. Phys. 10, 093005 (2008).

31. Guillamon, I. et al. Direct observation of melting in a two-dimensional superconducting vortex lattice. Nat. Phys. 5, 651-655 (2009).

32. Pfuner, F., Degiorgi, L., Baturina, T. I., Vinokur, V. M. \& Baklanov, M. R. Optical properties of TiN films close to the superconductor-insulator transition New J. Phys. 11, 113017 (2009).

33. Sacépé, B., Chapelier, C., Baturina, T. I., Vinokur, V. M., Baklanov, M. R. \& Sanquer, M. Disorder-induced inhomogeneities of the superconducting state close to the superconductor-insulator transition. Phys. Rev. Lett. 101, 157006 (2008).

34. Sacépé, B., Chapelier, C., Baturina, T. I., Vinokur, V. M., Baklanov, M. R. \& Sanquer, M. Pseudogap in a thin film of a conventional superconductor. Nat. Commun. 1, 140 (2010).

35. Baturina, T. I. et al. Superconducting phase transitions in ultrathin TiN films. Europhys. Lett. 97, 17012 (2012).

\section{Acknowledgements}

We are delighted to thank Yu. Galperin, A. Glatz, N. Kopnin, A. Mel'nikov, A. Bezryadin and B. Shapiro for illuminating discussions. The Laboratorio de Bajas Temperaturas is associated to the ICMM of the CSIC. This work was supported by the Spanish MICINN and MEC (Consolider Ingenio Molecular Nanoscience CSD2007-00010 programme, MAT2011-27553-C02, FIS2011-23488, FIS2010-21883 and ACI-2009-0905), by the Comunidad de Madrid through program Nanobiomagnet and by the Aragón regional Governement, by the Program 'Quantum mesoscopic and disordered structures' of the Russian Academy of Sciences, by the Russian Foundation for Basic Research (Grant No. 12-02-00152 and Grant No. 12-02-31302), and by the US Department of Energy Office of Science under the Contract No. DEAC02- 06CH11357.

\section{Author contributions}

R.C. and J.S. performed experiments on the wire and T.I.B. and A.Yu.M. performed experiments on the perforated film. D.A.N., A.K.G. and A.V.L. performed high-resolution and scanning electron microscopy of the film. R.C., T.I.B. and M.R.B. made the samples. T.I.B., J.M.D., M.R.I. and V.M.V. conceived the experiments. J.J.P. and V.M.V. made calculations. R.C., T.I.B., I.G., S.V., V.M.V. and H.S. analysed data and wrote the paper. All authors discussed the results and implications and commented on the manuscript at all stages.

\section{Additional information}

Supplementary Information accompanies this paper at http://www.nature.com/ naturecommunications

Competing financial interests: The authors declare no competing financial interests.

Reprints and permission information is available online at http://npg.nature.com/ reprintsandpermissions/

How to cite this article: Córdoba, R. et al. Magnetic field-induced dissipation-free state in superconducting nanostructures. Nat. Commun. 4:1437 doi: 10.1038/ncomms2437 (2013). 